\title{
INVESTIGACIONES
}

\section{Dispositivos de acogida para estudiantes extranjeros como plataformas de intervención formativa*}

\author{
Devices for welcoming foreign students as formative intervention platforms
}

\author{
René Valdés Morales ${ }^{a}$, Felipe Jiménez Vargas ${ }^{b}$ \\ María-Teresa Hernández-Yáñez $z^{c}$ Carla Fardella ${ }^{a}$ \\ ${ }^{a}$ Facultad de Educación y Ciencias Sociales, Universidad Andrés Bello. \\ revalmorales@gmail.com, fardellacarla@ hotmail.com \\ ${ }^{b}$ Pontificia Universidad Católica de Valparaíso. \\ felipe.jimenez@pucv.cl \\ ${ }^{c}$ Universidad de Santiago de Chile. \\ maitehernandezy@gmail.com
}

\section{RESUMEN}

La ausencia de un modelo gubernamental de acogida para la población escolar migrante ha obligado a las escuelas a rediseñar sus prácticas educativas, asumiendo de manera autodidacta y creativa el trabajo en torno a la diversidad cultural. Por esto, nos propusimos comprender cómo las escuelas han respondido a la llegada de estudiantes extranjeros y qué prácticas educativas han desplegado al respecto. Mediante un trabajo etnográfico de 2 años en 4 escuelas públicas con proyectos educativos con sello intercultural y alta matrícula de estudiantes extranjeros, se revela la existencia de 10 dispositivos de acogida que han permitido a las escuelas gestionar la inclusión educativa de estudiantes extranjeros. Analizamos dichos dispositivos en función de las dimensiones implicadas, grados de sofisticación y de las contradicciones que generan al interior de las escuelas, y discutimos sus alcances y contribuciones a partir de una experiencia de intervención formativa.

Palabras claves: prácticas escolares, inclusión, interculturalidad, migración.

\section{ABSTRACT}

The absence of a government model of reception for the migrant school population has forced schools to redesign their educational practices, assuming in a self-taught and creative way work around cultural diversity. Therefore, we set out to understand how schools have responded to the arrival of foreign students and what educational practices they have deployed in this regard. Through an ethnographic work of 2 years in 4 public schools with educational projects with intercultural stamp and high enrollment of foreign students, it is revealed the existence of 10 reception devices that have allowed schools to manage the educational inclusion of foreign students. We analyze these devices in terms of the dimensions involved, degrees of sophistication and the contradictions that they generate within schools, and we discuss their scope and contributions based on an experience of formative intervention.

Key words: school practices, inclusion, interculturality, migration.

\footnotetext{
* Financiamiento: Proyectos CONICYT/Fondecyt $\mathrm{N}^{\circ}$ 11150831, CONICYT/Fondecyt $\mathrm{N}^{\circ} 1190349$ y Anid/Fondecyt
} $\mathrm{N}^{\circ} 3200192$ 


\section{INTRODUCCIÓN}

La presencia de estudiantes extranjeros en Chile se ha convertido en un fenómeno que progresivamente ha ido impactando la estructura del sistema educativo y con ello el desafío de implementar una serie de adecuaciones en respuesta a las necesidades y contingencias. Durante el período 2015-2018 la matrícula de estudiantes inmigrantes se cuadriplicó ${ }^{1}$. Además, este incremento se refleja con mayor fuerza en establecimientos de administración pública, aumento que también se manifiesta en regiones y comunas que cuentan con una alta concentración geográfica de población migrante (Elige Educar, 2017). Los desplazamientos migratorios vividos en nuestro país durante los diez últimos años, muestran la llegada de nuevos colectivos de migrantes, como es el caso de la población haitiana y recientemente la población siria, formando parte de una demanda que también se evidencia en el ámbito escolar. Dichos requerimientos dan origen a otras necesidades educativas, como por ejemplo de tipo lingüísticas que traen como consecuencias barreras idiomáticas a las trayectorias estudiantiles, y con esto el desafío para las comunidades educativas de enfrentar nuevas problemáticas asociadas a la presencia de diversidad estudiantil. Además, a la fecha nuestro sistema educativo no ha propuesto formalmente ningún modelo explícito de acogida que permita favorecer la inclusión de estudiantes extranjeros. Esto dificulta la generación de cambios necesarios que garanticen que dicha inserción se efectúe desde un enfoque inclusivo y de acogida (Riedemann, 2017).

Sin embargo, el Ministerio de Educación consciente de las necesidades que viven las escuelas, impulsa una serie de orientaciones e indicaciones generales con el objeto de instalar un enfoque intercultural en las prácticas educativas. Pese a esto, las indicaciones ministeriales aún no cuentan con una validez empírica por parte de la investigación educativa en cuanto a su respuesta a los contextos locales y con eso a una mayor significación del trabajo con estudiantes extranjeros. Lo anterior ha generado que las escuelas frente a la necesidad de abordar el fenómeno migratorio diseñen e implementen desde su autoaprendizaje (Poblete y Galaz, 2017) una serie de acciones y dispositivos de acogida en apoyo a estudiantes extranjeros al interior de sus comunidades educativas.

Frente a esta realidad que protagonizan las escuelas, nos preguntamos de qué forma enfrentan la llegada de estudiantes extranjeros y qué mecanismos de acogida levantan para favorecer su inclusión educativa. Este artículo pretende responder a estas dos interrogantes que movilizaron nuestra investigación.

\section{DISPOSITIVOS DE ACOGIDA AL SISTEMA EDUCATIVO CHILENO}

Para aproximarnos al fenómeno de la acogida conviene hacernos previamente la pregunta acerca de qué es un dispositivo. Para ello nos apoyaremos en desarrollos propios de los estudios psicosociales del trabajo (Fardella y Carvajal, 2018; Sisto y Zelaya, 2013). Desde este campo de estudio y en base a la Teoría de Actor Red (Latour, 2008) se define un dispositivo como ensamblajes materiales y discursivos que intervienen en la acción local configurándose como objetos con agencia (Sisto y Zelaya, 2013). De esta manera un dispositivo tiene la capacidad de transformarse en un actor como tal, y por tanto, tiene

Matrícula se ha cuadriplicado de 30 mil a 130 mil estudiantes extranjeros en escuelas chilenas. 
la posibilidad de interactuar con otros actores e influir en las acciones de éstos. Como señala Harré (2002) hay dos características claves de los dispositivos: por un lado, que éstos pueden adquirir diferentes identidades dependiendo de cómo sean narrados por los actores que los usan; y por otro, que los dispositivos actúan y desarrollan diferentes roles y grados de actividad de acuerdo con las relaciones que establecen.

Desde esta óptica diferentes objetos materiales e inmateriales característicos del funcionamiento escolar: libros de clases, incentivos, protocolos de acción, planes de gestión, incluso programas como el de integración escolar; podrían ser considerados como actores educativos que tienen la capacidad de influir y determinar la actividad del sistema escolar. De allí nuestro interés en focalizarnos en el papel que juegan los diferentes dispositivos de acogida para estudiantes extranjeros.

Sobre la base de estas características centrales, entendemos los dispositivos de acogida como objetos y actuaciones educativas creadas a través del ensamblaje de recursos personales, didácticos, culturales y metodológicos, que se incorporan institucionalmente al funcionamiento escolar para organizar las intervenciones educativas orientadas a facilitar la inclusión de estudiantes de origen extranjero (Fernández y García Castaño, 2015; González, 2017; Jiménez, 2017; Simó, Pàmies, Collet-Sabé y Tort, 2014). Como demuestran los análisis internacionales sobre dispositivos de acogida (González-Falcón, Gómez-Hurtado y ÁlvarezDíaz, 2017), independiente de su naturaleza, propósito y ámbito de acción, todo dispositivo tiene su propia trayectoria vital y poner el acento en dichas trayectorias es clave para comprender cómo se insertan tales dispositivos en la arquitectura de la escuela y cómo este proceso de acoplamiento puede promover la reproducción y estancamiento o bien la expansión de la escuela como sistema de actividad (Engeström, 2007; Sannino, Engeström \& Lemos, 2016).

Entender por tanto los dispositivos de acogida como objetos y/o actuaciones educativas que emergen de manera autodidacta en las escuelas, así como analizar la forma en que éstos alteran e interpelan la actividad del sistema-escuela, se constituye a nuestro juicio en un ejercicio pendiente toda vez que la política educativa vigente no aporta elementos significativos para la toma de decisiones en materia de dispositivos de acogida.

\section{METODOLOGÍA}

Dado el objeto de estudio, optamos por un enfoque etnográfico secuencial (Díaz de Rada, 2013; Rockwell, 2009), específicamente, una etnografía de dispositivos (Sisto y Zelaya, 2013), cuya principal técnica de producción de datos fue el shadowing (Czarniawska, 2007; Pujadas, 2018), ya que se ha ido posicionando progresivamente como una de las estrategias etnográficas más promisorias en el estudio de las prácticas educativas (Earley, 2012; McDonald, 2005), Esto debido a que el shadowing, vale decir hacer sombra, estar cerca de alguien, algo o una acción durante un determinado período de tiempo (Fardella y Carvajal, 2018), otorga una importancia central al seguimiento situado y en tiempo real del objeto de estudio. Este formato etnográfico, a diferencia de otras modalidades tradicionales, permite acceder a espacios, conversaciones, procedimientos y narraciones que emergen durante la misma participación del investigador y no posteriormente, como suele ocurrir, por ejemplo, al momento de concertar entrevistas con determinados actores claves.

Participaron del estudio cuatro establecimientos educacionales: dos escuelas de la comuna de Estación Central y una escuela de Quilicura en la región metropolitana, y un 
instituto de enseñanza media de la cuarta de la región de Antofagasta. Las cuatro escuelas adscriben su proyecto educativo institucional a un sello inclusivo e intercultural y cuentan con una elevada presencia de estudiantes extranjeros, sobre el $40 \%$ de la matrícula. El trabajo de campo se prolongó desde mayo de 2017 hasta septiembre de 2018.

Para la producción de datos se privilegiaron las observaciones participantes y complementaron con entrevistas, grupos focales y el análisis documental. Todas estas técnicas se desarrollaron de modo secuencial (Voirol, 2013). Vale decir, en una primera fase nos insertamos en diferentes espacios escolares, formales como sala de clases e informales como comedores, patios y accesos). En la medida que fuimos identificando dispositivos claves, se agendaron entrevistas y grupos focales con actores claves. Finalmente se revisaron documentos institucionales (proyecto educativo, reglamentos internos, plan de convivencia, plan de apoyo a la inclusión, protocolos de acción). La tabla 1 resume la producción de datos realizada.

Tabla 1. Consolidado de producción de datos

\begin{tabular}{|l|c|c|c|c|c|}
\hline & Escuela 1 & Escuela 2 & Escuela 3 & Escuela 4 & Total \\
\hline Observaciones & 15 & 12 & 5 & 3 & 35 \\
\hline Entrevistas & 12 & 16 & 3 & 3 & 34 \\
\hline Grupos focales & 4 & 2 & 1 & 4 & 11 \\
\hline
\end{tabular}

Fuente Propia.

Para analizar utilizamos el análisis de contenido temático (Vásquez, 1996). Mediante una triangulación de técnicas (Flick, 2014) identificamos aquellos dispositivos relevantes con los cuales se establecieron las siguientes etapas de trabajo: (1) lecturas de datos, (2) codificación abierta, (3) categorización (4) triangulación, (5) reunión del equipo de investigación y (6) consenso de dispositivos relevantes.

Tanto para la inmersión etnográfica en general como para el desarrollo de las diversas técnicas específicas, se solicitaron previamente las autorizaciones al equipo directivo de las escuelas participantes, se incorporaron consentimientos informados a adultos y asentimientos informados a estudiantes, todos ellos visados por el comité de bioética de la universidad patrocinante. Una vez finalizadas las inmersiones etnográficas y analizados los resultados, se desarrolló en cada escuela una intervención formativa (Engeström, 2009; Erausquin y Dome, 2016) que a través de un taller permitió realizar discusiones colaborativas de los resultados presentados. Profundizaremos esto último a partir del concepto de trueque constructivo (León, 2007) en el apartado final de las proyecciones de nuestro trabajo.

\section{RESULTADOS}

Los dispositivos etnografiados son los siguientes: (1) Protocolo de acogida, (2) equipo bilingüe, (3) fiesta intercultural, (4) pauta observación con sello intercultural, (5), aula de 
acogida, (6) mediación escolar, (7), grupo diferencial (8) estudiantes tutores, (9) grupos interactivos y (10) cuota migrante. En razón del espacio disponible realizaremos una reseña de cada uno de ellos, en el orden en que fueron emergiendo de nuestro trabajo de campo.

\subsection{PROTOCOLO DE ACOGIDA}

El protocolo de acogida corresponde al primer dispositivo que nos interesó documentar y es junto a los grupos interactivos -como veremos más adelante- la excepción a la regla con relación a la naturaleza autodidacta que caracteriza los dispositivos de acogida. Corresponde a un dispositivo importado; de hecho es impulsado precisamente por profesionales de origen extranjero en las escuelas donde ha sido implementado. De hecho, el protocolo de acogida está ampliamente incorporado -y prescrito- en la mayoría de las escuelas públicas de las comunidades autónomas de España (Alegre, 2005; Generalitat de Catalunya, 2015). Podemos definirlo como un documento que reúne un conjunto de procedimientos y acciones orientadas a facilitar la matriculación de estudiantes extranjeros, enfocado principalmente al área administrativa. No obstante, algunas escuelas han incorporado acciones pedagógicas (como evaluación inicial a estudiantes extranjeros) como complemento al carácter orientador del dispositivo. Ya que a la fecha no existen instrumentos de gestión prescritos por las políticas educativas para estos fines, el protocolo surge como iniciativa de las escuelas para resolver sus propios desafíos y contradicciones (Engeström y Sannino, 2016). De esta manera, los protocolos de acogida, independiente de su naturaleza, funcionan como una herramienta que articula la actividad educativa de la escuela. En algunos casos contemplan la dimensión administrativa, lo curricular-pedagógico y el trabajo con las familias, mientras que otros protocolos se focalizan mayormente en apoyar los procesos de regularización de estudios y obtención de matrículas provisorias. En cualquiera de sus versiones, el propósito del protocolo es siempre orientar la acción educativa en torno a la llegada de estudiantes extranjeros. Nuestro trabajo etnográfico dejó en evidencia que el protocolo de acogida actúa tanto como un documento de consulta (objeto), pero a la vez un dispositivo que guía la acción (sujeto) que pone en funcionamiento el actuar de otros. Esto lo releva como un dispositivo omnipresente y orquestante con relación a otros dispositivos más específicos y de alcance más acotado. Muy gráfico fue la experiencia de uno de los investigadores del equipo quien durante una de las sesiones etnográficas y aprovechando la ausencia de la trabajadora social quien había redactado el protocolo, se acercó a la secretaria para preguntarle dónde podríamos encontrar el protocolo. Su respuesta no requiere mayor explicación: “... el protocolo está en todas partes” (notas de campo, investigador 1, escuela 1). La existencia de protocolos de acogida ya había sido previamente reportada por investigaciones en la materia (FUSUPO, 2017), incorporado más recientemente en documentos ministeriales (Mineduc, 2017) y presentado como desafío en la política nacional para estudiantes extranjeros (Mineduc, 2018).

\subsection{EQUIPO BILINGÜE}

Etnografiando el protocolo de acogida, aparece rápidamente en escena la existencia de una dupla de profesores haitianos, el equipo bilingüe, que realizan funciones de apoyo, interpretación y traducción a estudiantes haitianos que aún no manejan el idioma español en la escuela. Lo primero que llama nuestra atención es la diversidad de nombres que 
recibe: traductores, intérpretes, monitores, facilitadores, mediadores, profesores haitianos o simplemente, profesores de creol. El propósito general de su incorporación por parte del equipo directivo es minimizar al máximo la barrera idiomática. De esta manera, las tareas del equipo se encuadran principalmente en el área técnico-pedagógica. Una de sus principales características es su semejanza metodológica con los profesionales del programa de integración escolar: su forma de abordar la evaluación y los apoyos, el hecho de trabajar con ciertos estudiantes, apoyar en aula regular y aula de recursos, así como desarrollar adecuaciones curriculares. A diferencia del protocolo de acogida que por lo que pudimos constatar recibe una valoración discrepante por parte de sus usuarios/destinatarios, el equipo bilingüe corresponde a una iniciativa que, pese a sus debilidades y desafíos, es valorada positivamente por la mayoría de los actores que se benefician de su apoyo. Una nota de campo de uno de nuestros diarios transmite esto:

los profesores de la escuela 1 describen a los profesores haitianos como "apañadores", pues representan una forma de descompresión ante la gran cantidad de estudiantes haitianos. Al mismo tiempo, se manifiesta en sus relatos una forma afectuosa de relación, que radica principalmente en sentirse plenamente apoyados (notas de campo, investigador 2, escuela 1).

\subsection{FIESTA INTERCULTURAL}

La fiesta intercultural es quizás el dispositivo con mayor trayectoria en nuestro sistema educativo, de hecho, ya habíamos reportado su existencia en una investigación exploratoria previa en escuelas de la región metropolitana realizada en el período 2012-2013 (Jiménez y Fardella, 2015). Corresponde a una celebración que se realiza en las escuelas en la que se realizan diferentes actividades que permiten exponer y visibilizar la diversidad cultural existente. Si bien corresponden a espacios de recreación no formal que promueven las interacciones interculturales (FUSUPO, 2017), se constituyen a nuestro juicio en espacios que facilitan y vehiculizan aprendizajes no formales. Nuestros entrevistados utilizan diferentes formas para referirse a ella: algunos refieren a una fiesta intercultural o fiesta de la interculturalidad, mientas otros a un festival o incluso a una feria intercultural (notas de campo, investigador 2, escuela 1). Aun cuando la fiesta intercultural comenzó como una iniciativa para conmemorar el 12 de octubre, en la actualidad funciona en algunas escuelas como un proyecto que permite poner en diálogo la convivencia escolar, el trabajo pedagógico e incluso el desarrollo de continuidades familia-escuela. Al igual que el protocolo de acogida, la fiesta intercultural se ha ido posicionando progresivamente como un dispositivo de segundo orden, vale decir un dispositivo que permite orquestar otros de menor complejidad. La siguiente viñeta tomada de una conversación con una docente lo demuestra:

Nosotros no celebramos el 18 de septiembre, es decir, lo celebramos chiquitito, bailamos un pie de cueca, una empanada (...) pero sí celebramos el día de las Américas, el día de la raza, que se llamaba antes. Claro, por ahí, nosotros hacemos una fiesta que se llama "Latinoamérica celebra a Chile" o "Chile a Latinoamérica celebra" una cuestión así. Y esa es una fiesta intercultural pero inmensa, inmensa, que cada curso toma un país de Latinoamérica (...) y al azar, tení que preparar la bandera, los trajes, la comida y entre que vamos haciendo eso vamos hablando de la 
historia, y vamos haciendo posters, y vamos haciendo banderas con pedacitos de papel, y vamos haciendo banderitas (...) Esto primero partió como un festival intercultural para incorporar a las distintas culturas presentes acá en la escuela, y ahora quedó en el PME, quedó instalado (profesora, escuela 1).

Como pudimos constatar el año 2016 a partir de un mapeo en torno a la investigación académica sobre migración y escuela realizada en Chile (Jiménez et al., 2017), las fiestas interculturales corresponden al dispositivo más ampliamente instalado en nuestras escuelas, y por lo tanto, a nuestro juicio el más intuitivo con relación a la gestión de la diversidad cultural, pero no por eso menos complejo que otros más idiosincráticos.

\subsection{PAUTA OBSERVACIÓN CON SELLO INTERCULTURAL}

A medida que avanza nuestra inmersión etnográfica nos enteramos de la existencia de una pauta de observación de aula diseñada e implementada por el equipo directivo, como estrategia curricular para evaluar las prácticas docentes. La jefa de la unidad técnico-pedagógica de la escuela la explica como "un acompañamiento" (notas de campo, investigador 3, escuela 1), que tiene como intención apoyar el trabajo del docente desde la perspectiva de la mejora continua. En sus propias palabras, "es como una evaluación interna que ahora se le llama acompañamiento de aula. Porque si bien sigue siendo una evaluación, el objetivo no es evaluarlos sino más bien retroalimentar estos aspectos más débiles en el docente". (jefa UTP, escuela 1). Ahora bien, ¿qué significa que tenga un sello intercultural? Es una pregunta que nos planteamos como equipo y que intentamos responder en nuestras siguientes sesiones etnográficas. La pauta evalúa la didáctica, el clima de aula y las interacciones pedagógicas. Es justamente en esta última dimensión donde se pone en juego el sello intercultural de una clase, velando por una comunicación e interacción cultural horizontal entre diferentes grupos humanos, con costumbres y experiencias de vidas cotidianas diversas, de modo que no prevalezca una jerarquización cultural, sino desde un espacio compartido y un ambiente de reconocimiento y participación. A partir de dicha estrategia educativa se entrega una retroalimentación del trabajo, y en forma conjunta, se convierte en un espacio de autoevaluación y visualización de sus propias prácticas en el marco de una mejora continua y transformación de una escuela que acoge a estudiantes con diversidad étnica y lingüística.

Ahora bien, como hemos podido documentar la implementación de la pauta de observación no ha estado exenta de dificultades. La introducción de este dispositivo en el entramado del sistema escuela activa diferentes contradicciones internas y, por tanto, diferentes procesos de resistencia. Esto ya que la introducción -no voluntaria, por ciertode la observación de aula, que supone al mismo tiempo la evaluación del desempeño docente, supone cambios tanto en la propia comunidad docente así como en la división del trabajo de sus integrantes. Respecto a esto último, es evidente que evaluar el sello intercultural de una clase supone para el docente a cargo incorporar nuevos elementos en el diseño, materiales, nuevas metodologías de evaluación. Todo ello recordemos, en un contexto como telón de fondo basado en la precariedad del sistema educativo público:

Ha implicado una revisión tanto hacia arriba como hacia abajo, ¿por qué? Porque lo primero es decir, y esto lo he escuchado, "es que los niños extranjeros no entienden nada y bajan el nivel". ¿Vale? Pero también provoca que tú te has acostumbrado a ser 
un profesor que llega a clases, cumples con tu planificación y me voy. Y bueno pues de alguna forma yo creo que lo que ha provocado [la pauta] es recuperar un poco la magia en la creatividad del docente. Pero ha provocado muchas tensiones, porque no nos olvidemos que los docentes de escuelas municipales cobran poco dinero, trabajan muchas horas, tienes cursos de 45 niños, $i 45$ ! (trabajadora social, escuela 1).

\subsection{AULA DE ACOGIDA}

El aula de acogida es un dispositivo cuya forma de aparición es particularmente interesante ya que, si bien responde a la creatividad de las propias escuelas, es junto a los protocolos un dispositivo fuertemente institucionalizado en algunos países con tradición en la escolarización de estudiantes extranjeros (Alegre, Benito y González, 2012; Arroyo y Torrego, 2012; Jiménez, 2017). En su versión local, es un espacio físico de trabajo conducido por un profesor de nacionalidad haitiana, un aula especialmente diseñada para el aprendizaje lingüístico, alternativo al aula ordinaria y/o de referencia de los estudiantes. De acuerdo con la literatura especializada corresponde a una de las tres principales prácticas de agrupamiento de estudiantes para responder a sus necesidades de aprendizaje, el streaming o agrupamientos homogéneos (Flecha, 2015; Valls \& Kyriakides, 2013). En este formato, el profesor realiza funciones de apoyo, interpretación y traducción a los estudiantes haitianos que aún no manejan el idioma español. A diferencia del equipo bilingüe, las ayudas pedagógicas se realizan como pudimos observar, fuera del aula ordinaria. Si como señalamos anteriormente, el trabajo del equipo bilingüe es equiparable al trabajo de los equipos PIE, el trabajo en el aula de acogida es comparable al de los paulatinamente extintos grupos diferenciales: trabajar por grupos, bajo ciertas edades o cursos, entre 60 y 90 minutos por semana, siendo su propósito principal el aprendizaje del español. Pese a ello y como pudimos observar mediante algunas de nuestras sesiones de shadowing al interior del aula de acogida, no siempre existía un plan de desarrollo/trabajo individual vinculado a los aprendizajes esperados de acuerdo al nivel y/o curso de los estudiantes participantes. Esto nos lleva a pensar que la reciente puesta en marcha del dispositivo y su corta trayectoria de implementación lo hacen un dispositivo aún poco formalizado e instituido en la red de gestión de la diversidad del sistema-escuela. Esta falta de articulación aula de acogida-aula ordinaria genera efectos pedagógicos indeseados que, sin embargo, ya han sido detectados por la propia escuela y considerados para la implementación de cambios de mejora. Respecto a la necesaria vinculación pedagógica del aula de acogida, la jefa UTP de la escuela 2 señala:

Sabes que en algún momento, eh, manejamos un poco esa, esa alternativa pero la descartamos pensando que era muy exigente para y que no era justo con Rosita lo sentimos un poco, pedirle que domine todo los aprendizajes de primero a octavo (...) cómo le vamos a pedir a Rosita si ni siquiera nosotros, porque nosotros nos establecemos en un ciclo, a veces en cursos primero-segundo, tercero-cuarto y nos hacemos especialistas en esos niveles y le vamos a pedir a ella que maneje de primero a octavo todo, lenguaje, matemáticas, historia y ciencias naturales, cuatro asignaturas básicas. Y por eso un poco lo descartamos también (Jefa UTP, escuela 2).

De lo anterior se observa que la escuela ha encontrado uno de los principales problemas que es la sobrecarga hacia un solo profesional y ha avanzado con una posible solución: 
fraccionar o distribuir los estudiantes extranjeros sin dominio de la lengua vehicular en varios profesionales. Si pensamos en los dispositivos anteriores, la escuela ha encontrado la salida a la contradicción que la vinculación pedagógica plantea, precisamente avanzando hacia la creación de un equipo bilingüe que pueda hacerse cargo del aula de acogida. Esto como veremos con mayor detenimiento en el apartado de discusiones, es una muestra de cómo los diferentes dispositivos de acogida más allá de sus contradicciones internas tienen la capacidad de generar aprendizajes expansivos (Sannino, Engeström \& Lemos, 2016), en este caso en torno a la gestión de la diversidad cultural.

\subsection{MEDIADORA ESCOLAR}

La mediadora escolar emerge en el escenario escolar de una de nuestras escuelas participantes para dar respuesta a los conflictos interculturales que comenzaron a proliferar entre estudiantes a propósito del aumento de la matrícula de estudiantes extranjeros, así como de la consolidación de nuevas nacionalidades. Como hemos podido documentar, es el único dispositivo orientado exclusivamente al área de la convivencia escolar y de los climas de aula. Pese a lo anterior, no es un dispositivo que emerge a propósito de estos conflictos interculturales, sino más bien que se reinventa para dar respuesta a éstos.

La mediadora es una profesional que cumple labores ligadas a orientación escolar al interior de la escuela. Su rol se origina específicamente desde las demandas de profesores por contar con una persona que se hiciera cargo de los conflictos por lo que tiene múltiples roles: enseñar la resolución pacífica de conflictos, fomentar la comunicación efectiva y promover la reflexión entre los estudiantes. Con la llegada de estudiantes extranjeros sus roles incluyen la prevención de problemas de discriminación y promover la integración cultural. En palabras de la propia mediadora:

Porque a veces a mí me llegan derivaciones por los profes, los profesores me dicen “oye Sandra, mira, no sé”... ponle en ese caso fue al sexto. Y la... estaba de jefa la profesora Marta, entonces ella "Sandra, mira, tengo este problema, yo lo observo en la sala de que tengo una división que es chilenos pa' un lado, haitianos pa'otro (...) Y no se hablan, no di-, como... ay, como no pases pa' allá porque es zona haitiana y esta es zona chilena”, una cosa así, entonces me dijo como “¿qué podemos hacer? porque no puede ser que el curso esté así, desunido y todo”. Entonces ella habla conmigo y ya, voy a preparar algo, voy a hacer un tallercito, una charla (mediadora, escuela 2).

Esta viñeta nos muestra en este caso que la reorganización del propósito del dispositivo no es interno sino que proviene de las demandas de sus principales usuarios, en este caso profesores que requieren apoyo en el mejoramiento de las relaciones humanas entre estudiantes. Esto contrasta con los procesos de cambio de otros dispositivos, el protocolo de acogida o los grupos interactivos, que se transforman movilizados por causas y tensiones internas.

\subsection{GRUPO DIFERENCIAL CON FOCO MIGRANTE}

El grupo diferencial es un dispositivo complementario al aula de acogida, ya que tiene por objetivo apoyar los procesos de lectura y escritura en primer y segundo año básico de estudiantes haitianos. En cuanto al origen, el equipo directivo de una de las escuelas 
participantes lo explican como un "plan de contingencia", ya que es una estrategia que permite trabajar con aquellos estudiantes que no califican con los criterios para ingresar al PIE. En definitiva, es un dispositivo que expande los beneficios para estudiantes extranjeros que habitualmente quedan fuera de este programa.

Aun cuando es un dispositivo que no vimos en funcionamiento en nuestro trabajo etnográfico, de las entrevistas pudimos rescatar que participan profesores de primer y segundo año, una educadora especial, la profesora de creole y jefa de UTP. El grupo presenta una metodología de trabajo mixta: de forma individual y grupal y de inserción en aula y en aula de recursos. La profesora diferencial ingresa al aula y se focaliza en aquellos estudiantes que presentan problemas para adquirir el proceso lector. Cuando saca a los alumnos del aula, realiza una metodología de trabajo grupal con foco en los aprendizajes curriculares más descendidos.

\subsection{ESTUDIANTES TUTORES}

La existencia de estudiantes tutores es el único dispositivo presente en las cuatro escuelas participantes de nuestro estudio. Es además uno de los dispositivos de más pronta implementación debido a la urgencia que plantean las barreras lingüísticas. Si las fiestas interculturales son el dispositivo más intuitivo para la generalidad de las escuelas, los estudiantes tutores es el dispositivo más intuitivo para la especificidad de escuelas con barreras idiomáticas. En la mayoría de las escuelas es definido como una estrategia salvavidas: frente a la falta de profesionales de apoyo lingüístico, una alternativa razonable es recurrir al apoyo de estudiantes bilingües (lengua minoritaria y lengua vehicular).

En el caso de la escuela 3, los estudiantes tutores tiene un rol más sistematizado y por tanto más institucionalizado debido a que, como pudimos constatar en nuestro trabajo de campo, la transformación de la escuela hacia una comunidad de aprendizaje ha supuesto incorporar activamente a los estudiantes en las diferentes funciones educativas, incluyendo la gestión y las labores de apoyo docente. A pesar de lo anterior y al igual que con la mediadora escolar, los estudiantes tutores es un dispositivo al que sólo pudimos acceder vía relatos y no verlo en funcionamiento.

\subsection{GRUPOS INTERACTIVOS}

El grupo interactivo es también un dispositivo importado y de muy reciente incorporación en nuestras escuelas. Nos pareció un dispositivo muy interesante de incorporar en nuestra investigación ya que no surge para dar respuesta a las necesidades de estudiantes extranjeros, pero tampoco es su fin ulterior. Está orientado a todos los estudiantes, independiente de su nacionalidad, y para dar respuesta a todo tipo de necesidades educativas, no sólo las lingüísticas. Parafraseando a sus impulsores, corresponde a una actuación educativa de éxito que promueve la inclusión (Flecha, 2015; Valls, Buslón \& López, 2016). Lo anterior no quita, como nos advirtió el director de la escuela 3 en una de nuestras primeras visitas, que se haya mostrado como una promisoria estrategia para justamente favorecer el aprendizaje lingüístico de estudiantes extranjeros; a tal punto, que se haya extrapolado su uso a otros cursos -más allá del piloto original-y a otras asignaturas.

Si bien comparte el mismo propósito de adquisición del idioma español que otros dispositivos, como las aulas de acogida, difiere respecto a la forma de organización 
y principalmente del agrupamiento de los estudiantes, en la medida que privilegia el aula ordinaria, así como el trabajo colaborativo en grupos heterogéneos como principio metodológico de los procesos de enseñanza y aprendizaje. Como pudimos vivenciar de nuestra participación en una clase de matemáticas, el aula está compuesta por diferentes zonas de trabajo y el profesor está acompañado por un grupo de monitores/ayudantes (el investigador fue uno de ellos). Los estudiantes son distribuidos en cada uno de estas zonas de trabajo bajo un principio de heterogeneidad, con lo cual en cada grupo resultante hay mujeres y hombres, estudiantes autóctonos y extranjeros, así como estudiantes con diferentes niveles de dominio del contenido de lo que se va a realizar, en este caso ejercitar las operaciones matemáticas básicas. Por un espacio de 15 minutos, cada grupo acompañado por un monitor deberá realizar una tarea específica (guía de trabajo) que contempla actividades individuales como grupales. El monitor más que centrarse en entregar una ayuda técnica respecto a la tarea, cumple la función de dinamizar las interacciones entre los miembros del grupo, de manera que todos puedan interactuar con sus pares, poniendo especial énfasis en aquellos estudiantes con un bajo dominio de la lengua vehicular. Mientras cada grupo trabaja en resolver la tarea, el docente a cargo aprovecha de ir monitoreando el trabajo, poniendo especial énfasis en entregar ayudas adicionales a aquellos estudiantes que lo necesitan. Cumplido el tiempo el grupo se desplaza hacia la siguiente estación de trabajo, y con una nueva actividad y un nuevo monitor, que cumple el rol de facilitador de dicha estación educativa.

El grupo interactivo es también un buen ejemplo para mostrar cómo un dispositivo de acogida se puede presentar como una oportunidad de aprendizaje expansivo para el sistema-escuela y cómo la creatividad puede jugar un rol clave en la resolución de las tensiones que ponen en riesgo la estabilidad de los dispositivos. Esto ya que como nos relataron los participantes de la escuela 3 y comprobamos en primera persona, el éxito de los grupos interactivos pasa por la existencia del equipo de monitores que sostienen la actividad. Pero contar con un equipo estable de monitores es justamente una de las principales amenazas a la sostenibilidad del proyecto, toda vez que contar con profesionales disponibles en la escuela, así como incorporar padres y madres como monitores, se estaba volviendo algo cada vez más incierto. Es ahí donde la escuela toma en consideración la positiva experiencia de los estudiantes tutores y se plantea la posibilidad de probar un nuevo formato de monitores: los propios estudiantes de cursos superiores. Es así como la encargada del dispositivo nos narra que pudieron resolver uno de los principales nudos críticos de su diseño, pero no menos importante, expandieron su manera tradicional de considerar el rol de los estudiantes, mostrando que éstos eran capaces de desarrollar funciones habitualmente propias de personas adultas e idealmente profesionales. La escuela tuvo la oportunidad de construir una imagen de estudiante cualitativamente diferente a la anterior, imagen que naturalmente dio lugar al desarrollo de nuevas prácticas al interior de la escuela, impensadas con la antigua concepción de estudiante.

\subsection{CUOTA MIGRANTE}

Finalmente, el más reciente de los dispositivos etnografiados es la denominada cuota migrante. Emerge como una forma de visibilizar a los estudiantes extranjeros y, por tanto, favorecer su participación en espacios de institucionalidad: tanto la directiva de los cursos, así como la asamblea general de estudiantes deben tener entre sus miembros alumnos 
extranjeros. Por ejemplo, en el caso de la escuela 4 de nuestro estudio, el presidente del centro de estudiantes es un estudiante colombiano. Como nos contaron los propios estudiantes en los grupos focales, es algo inédito en la historia del establecimiento. La institución valora esta práctica pues considera que es una señal que no solo fomenta participación, sino que contribuye a crear una cultura escolar forjada en base al reconocimiento y la representación cultural al interior del instituto, dos elementos claves para promover la justicia social en el ámbito educativo (Fraser, 2011).

\section{DISCUSIÓN Y REFLEXIONES FINALES}

Como hemos intentado mostrar, los diferentes dispositivos expuestos varían entre sí en función de diversos criterios. Para efectos de este trabajo son especialmente relevante tres de ellos: en primer lugar, varían en función de su propósito y por lo tanto, de las dimensiones educativas puestas en juego; en segundo lugar, varían en función de sus principales destinatarios así como del grado de sofisticación de estos dispositivos; y finalmente, varían de acuerdo al tipo y profundidad de las tensiones y contradicciones que provocan al interior del sistema-escuela con su puesta en marcha. Discutiremos a continuación los dos primeros y en un apartado aparte el último de estos criterios.

Con relación a los propósitos y dimensiones educativas implicadas podemos observar que, haciendo una división gruesa entre el ámbito pedagógico, el ámbito convivencial y el ámbito de las relaciones familia-escuela y el ámbito de la gestión escolar, la gran mayoría de los dispositivos (equipo bilingüe, pauta de observación, aula de acogida, grupo diferencial y estudiantes tutores) están orientados a responder a las necesidades pedagógicas de los procesos de enseñanza y aprendizaje. Por el contrario, solo dos de ellos (mediadora y cuota migrante) se ponen al servicio del mejoramiento de la convivencia escolar. Los dispositivos restantes (protocolo, fiesta intercultural y grupos interactivos) son más bien dispositivos multipropósito y aportan en consecuencia a más de una de las dimensiones señaladas.

Si tomamos como ejemplo el protocolo de acogida, dispositivo que con mayor rapidez se ha ido masificando y que actualmente existe en cerca de un $40 \%$ de las escuelas según los resultados del primer censo docente sobre migración (Fundación Interhumanos, 2018), podemos ver que aun cuando es un dispositivo orientado principalmente a organizar administrativamente la gestión de la diversidad cultural, contempla no obstante acciones para facilitar los procesos de enseñanza-aprendizaje así como recursos para promover un enfoque intercultural de la convivencia entre pares. A su vez, en la medida que incorpora procedimientos para la matriculación es también un dispositivo que favorece el desarrollo de continuidades en las expectativas y metas entre la escuela y las familias inmigradas (Lamas y Lalueza, 2016).

Es muy útil además observar globalmente el proceso evolutivo de los diferentes dispositivos, la secuencia en la que van apareciendo, ya que ilustra la relevancia de cada una de las dimensiones en el tiempo. Es así que si hacemos un análisis desde el presente hacia el pasado se observa que la mayoría de los nuevos dispositivos se ubican en el ámbito pedagógico, justamente porque ha sido el ámbito de mayor tensión a propósito del cambio de los patrones migratorios (Estefoni y Bonhomme, 2014) en el ámbito escolar. La llegada de estudiantes con lenguas diferentes al español ha empujado a las escuelas a implementar iniciativas como los equipos bilingües, el aula de acogida o revivir antiguos 
dispositivos como el grupo diferencial para enfrentar las barreras idiomáticas antes inexistentes. Situación diferente es la del ámbito de la convivencia donde más que generar nuevas iniciativas, la respuesta de las escuelas ha sido más bien rediseñar y acomodar los dispositivos existentes a nuevas demandas; el caso de la mediación escolar es el caso más representativo de dicho funcionamiento.

Con relación al nivel de sofisticación pudimos observar que hay una gran dispersión: dispositivos valorados de forma etic y emic (Dietz, 2011) como altamente complejos y dispositivos que por su simpleza prácticamente no ocupan un lugar preponderante en los relatos de los actores educativos; son descritos como dispositivos que fluyen con autonomía. Ejemplos de dispositivos que fluyen son la pauta de observación, la cuota migrante, las fiestas interculturales, el protocolo de acogida. Por el contrario, dispositivos altamente complejos son el equipo bilingüe, el aula de acogida o los grupos interactivos, ya que requieren una atención constante, consumen recursos -humanos, materiales-, demandan una gestión y una toma de decisiones permanente. Si tomamos como ejemplo el equipo bilingüe podemos notar que no sólo implica incorporar un recurso adicional al trabajo de aula, sino que implica en primer lugar decidir a qué cursos/niveles se asignará el recurso, en qué asignaturas y a quiénes del conjunto de estudiantes extranjeros estarán dirigidos los apoyos; segundo, qué rol tendrá el facilitador lingüístico y qué apoyos ofrecerá, lo que implica articular su trabajo con el del docente a cargo de la clase. Si se opta por el tradicional trabajo en torno a un dossier individualizado, por ejemplo, cómo y quién será el encargado de evaluar dicho trabajo y qué vinculación tiene dicho aprendizaje con lo que está ocurriendo como telón de fondo en el aula. Como se puede observar, hay iniciativas que requieren una multiplicidad de decisiones muchas de ellas de gran complejidad debido al consenso que debe haber a su base y que supone articular las necesidades del propio docente, de la unidad técnico pedagógica, de los apoderados, entre otros.

Lo anterior nos lleva al último aspecto de nuestro análisis que, en estrecha vinculación con el anterior, tiene que ver con el tipo y profundidad de las tensiones y contradicciones que justamente los dispositivos sofisticados generan al interior de la escuela como sistema de actividad (Engeström, 2009).

\subsection{TENSIONES, NUDOS CRÍTICOS Y/O CONTRADICCIONES}

Como habíamos adelantado, todos los dispositivos anteriormente mencionados -tanto por su contenido o por su forma- producen tensiones y contradicciones para la organización escolar. Estas tensiones han sido reportadas tanto por los mismos actores escolares como también en nuestro rol de investigadores en el trabajo de campo realizado en las 4 escuelas. El análisis de estas tensiones y contradicciones es clave, toda vez que permite hacer el ejercicio de discriminar entre todos los dispositivos existentes, aquellos que presentan mayor potencialidad para generar aprendizajes expansivos en las escuelas. Como presentar los análisis de los 10 dispositivos resulta inviable por razones de extensión, privilegiaremos uno de los de mayor grado de sofisticación y que mejor transmite nuestra apuesta por preponderar las propiedades expansivas de los dispositivos de acogida: el aula de acogida. Recordemos que el aprendizaje expansivo alude al proceso por el cual un sujeto transforma o modifica su repertorio cultural para actuar de un modo diferente al habitual (Engeström, 2007), expansión que se logra resolviendo las tensiones y contradicciones internas a las que se enfrenta dicho sujeto. 
En el aula de acogida se pueden detectar dos tensiones principales. La primera guarda relación con el foco de trabajo. Según docentes y directivos, el trabajo del aula de acogida está vinculado más al idioma y poco a los aprendizajes curriculares. Esto hace que mientras un grupo de estudiantes avanza en el dominio de las competencias lingüísticas necesarias para seguir con normalidad los procesos de enseñanza y aprendizaje, retrocede en la adquisición de las competencias curriculares que están siendo trabajadas en el aula de referencia o aula ordinaria. Esta contradicción interna del dispositivo plantea una segunda tensión más amplia, en la medida que para garantizar la inclusión de ciertos estudiantes se utilizan paradójicamente espacios educativos segregados. Cómo romper este círculo de inclusión excluyente (Betancur, 2016; Gentili, 2011) se constituye como uno de los principales desafíos del dispositivo y por tanto de la escuela que lo implementa. Como mencionamos en el apartado descriptivo, la escuela 2 ha evaluado diversas fórmulas para responder a este desafío, desde dotar al dispositivo de más recursos profesionales o capacitar a los profesores extranjeros en el currículum escolar chileno. La pregunta que toda escuela se hace frente a estas interrogantes es siempre la misma: ¿cuál de estas alternativas es la mejor y cómo avanzar en el rediseño del dispositivo? Como hemos podido evidenciar en nuestro trabajo de campo, las respuestas no siempre están al alcance de la mano. Prueba de esto es el comentario de la jefa UTP de una de las escuelas participantes cuando nos dice:

(...) este año ha aumentado considerablemente la matrícula de estudiantes migrantes, en su mayoría haitianos, por lo que espero con ansias la retroalimentación que nos puedan entregar [aludiendo al equipo de investigación] ya que no tenemos tiempo para seguir ensayando estrategias y metodologías, debemos dar ya con la forma más adecuada de responder a estos niños y niñas (jefa UTP, escuela 2).

\subsection{PROYECCIONES: UN NUEVO ESTATUS PARA LA INVESTIGACIÓN EDUCATIVA}

La investigación académica en educación atraviesa un momento crucial: seguir aferrada a su rol tradicional de describir la realidad educativa o avanzar hacia un nuevo estatus donde contribuya a la transformación de dicha realidad educativa. La principal dificultad de nuestra investigación por contar con escuelas participantes refleja el agotamiento y pérdida de sentido para las escuelas de este tipo de investigación. Es en este escenario que la idea de trueque constructivo se presenta como una oportunidad para superar las limitaciones de la actual forma de producir conocimiento (León, 2007).

Como una forma de ilustrar esta nueva perspectiva, la experiencia de nuestro equipo de investigación que lejos de constituir una práctica consolidada, puede servir de ejemplo para graficar la contribución que el ámbito académico puede hacer hacia a las escuelas participantes de sus investigaciones.

Desde los desarrollos contemporáneos de la teoría de la actividad en educación (Kontos, 2016; Larripa y Erausquin, 2008), implementamos en una de las escuelas participantes una versión piloto y exploratoria de intervención formativa (Engeström, 2011). Para esto propusimos al equipo directivo una primera fase compuesta por 4 sesiones. En la primera de ellas trabajamos con el equipo de gestión ampliado y el consejo de profesores, espacio en el que presentamos los resultados generales de nuestra investigación -la descripción de los dispositivos aquí expuesta- y profundizamos en aquellos existentes en esa escuela. A su vez, planteamos nuestras inquietudes respecto a las tensiones y contradicciones que 
detectamos en dichos dispositivos. Invitamos finalmente en un plenario a proponer vías posibles de solución a dichas tensiones y contradicciones.

Pese a nuestras buenas intenciones como equipo, la respuesta a nuestra invitación fue bastante pasiva e indiferente. Claramente la escuela esperaba una devolución más propositiva y directiva a fin de cuentas en cómo mejorar las dificultades que enfrentaban los dispositivos implementados. Como una forma de corregir nuestra primera sesión, sugerimos una segunda sesión de trabajo, esta vez únicamente con el equipo de gestión ampliado. En ella retomamos las dificultades de algunos dispositivos seleccionados -aula de acogida y mediación escolar- discutimos algunas sugerencias de mejora incorporado además la experiencia de las otras escuelas en la implementación de ciertos dispositivos. Como resultado de ese trabajo surgió la iniciativa de trasladar simbólicamente el aula de acogida al aula ordinaria, de manera que el aprendizaje de la lengua vehicular se pudiese realizar simultáneamente al trabajo en torno a los contenidos disciplinares de las asignaturas. Sugerimos finalmente a la escuela que en una tercera sesión de trabajo interno pudiesen valorar la propuesta y nos ofrecimos finalmente para colaborar en el diseño del traslado del aula de acogida. Actualmente estamos a la espera de una cuarta sesión para reencontrarnos y diseñar conjuntamente un plan de trabajo para implementar los cambios y mejoras.

De esta acotada experiencia de intervención formativa hemos podido extraer no obstante tres enseñanzas profundamente significativas para nuestro trabajo de investigación. En primer lugar, que no basta con visibilizar las contradicciones del sistema de actividad o de alguno de sus componentes para que éstas se vuelvan automáticamente significativas para sus participantes; se hace necesario que dichas contradicciones sean experimentadas y luego reveladas y reconvertidas a conflictos personales (Engeström y Sannino, 2016). De lo contrario se corre el riesgo de que una tensión, paradójicamente, se considere de importancia, pero poco relevante de resolver. Ejemplo de esta paradoja es el hecho que para nuestro equipo era altamente preocupante -y tensionante- que el aula de acogida no contemplara una evaluación inicial, de proceso y final del dominio lingüístico de sus participantes, preocupación que trasformamos en sugerencia en el plenario de la primera sesión. Pese a esto no hizo eco entre los profesionales de la escuela, lo que supone por cierto un primer desafío a la hora de pensar una futura instancia de devolución de resultados o de trabajo colaborativo en torno a estos. En segundo lugar, que no basta con entregar un conjunto de recomendaciones sin los apoyos o el andamiaje necesario para poner en marcha los cambios recomendados. Es necesario que las recomendaciones estén dentro de la zona de desarrollo próximo del sistema de actividad (Daniels, 2003) pero al mismo tiempo que existan los procesos de mediación que favorezcan el tránsito entre zonas. La metáfora de los tres cuartos planteada por Chaiklin \& Hedegaard (2013), advierte que los agentes educativos no cuentan con todos los conocimientos, habilidades o procedimientos necesarios para producir por sí mismos y con autonomía disciplinar, las transformaciones expansivas; el insight (cuarto restante) se logra trabajando conjuntamente con otros profesionales. Por esto es clave generar agencias inter-profesionales (Yamazumi, 2009) donde converja todo el saber acumulado de la propia escuela y sus profesionales y el saber académico acumulado a través de la investigación educativa. En tercer lugar y para finalizar, que la entrada de un equipo de investigación al espacio social de la escuela siempre supone independiente del grado de profundidad de su trabajo, la incorporación de un nuevo sistema de actividad (Erausquin y Dome, 2016); incorporación que implica un diálogo inter-sistemas que impacta y puede producir cambios en la comunidad que 
conforman dicho sistemas, pero principalmente en la división del trabajo que realizan respectivamente. Resolver las tensiones y contradicciones que plantean los dispositivos de acogida ya no es una tarea exclusiva de la escuela, sino que debe ser compartida, negociada y distribuida con el equipo que lidera la intervención-formativa.

Un nuevo estatus para la investigación académica en educación sería sin duda una enorme contribución a la actual política nacional de estudiantes extranjeros, que como hemos señalado al comienzo de este trabajo, tiene la deuda de incorporar el trabajo interprofesional para responder a los desafíos que la escolarización de estudiantes extranjeros plantea a nuestras escuelas públicas.

\section{REFERENCIAS BIBLIOGRÁFICAS}

Alegre, M. (2005). Educació i imigració: l'acollida als centres educatius. Barcelona: Editorial Mediterránea.

Alegre, M., Benito, R. y González, S. (2012). Experiencias escolares iniciales del alumnado inmigrado: comienzos que marcan. Educación XX1, 15(2), 137-158.

Arroyo, M. y Torrego, L. (2012). Estudio comparado entre las medidas de atención lingüística para el alumnado inmigrante en las diferentes comunidades autónomas españolas, Revista Electrónica Interuniversitaria de Formación del Profesorado, 15(3), 35-44.

Betancur, J. (2016). Una inclusión excluyente: reconfigurando la inclusión en la escuela. Revista de Investigaciones UCM, 16(27), 178-188. doi: http://dx.doi.org/10.22383/ri.v16i1.69.

Chaiklin, S. y Hedegaard, M. (2013). Cultural-historical theory and educational practice: Some radical-local considerations. NUANCES: Estudos sobre Educação, 25(1), 30-44.

Czarniawska, B. (2007). Shadowing and Other Techniques for Doing Fieldwork in Modern Societies. Malmö: Liber.

Daniels, H. (2003). Vigotsky y la pedagogía. Buenos Aires: Paidós.

Díaz de Rada, A. (2013). Etnografía de la escuela más allá de la etnografía y de la escuela: tensiones disciplinares y aplicabilidad de los saberes etnográficos. Educación y Futuro, Revista de investigación aplicada y experiencias educativas, (29), 13-39.

Dietz, G. (2011). Hacia una etnografía doblemente reflexiva: una propuesta desde la antropología de la interculturalidad, Revista de Antropología Iberoamericana, 6(1), 3-26.

Earley, P. (2012). Observation methods: learning about leadership practice through shadowing. Journal of Educational, cultural and Psychological studies, 3(6), 15-31. doi: http://dx.doi.org/10.7358/ ecps-2012-006-earl.

Elige Educar. (2017). Aula Maestra. Un nuevo desafío para la educación chilena: ser un país de migrantes. Santiago: Elige Educar. Recuperado de https://eligeeducar.cl/wp-content/ uploads/2017/07/aulam_10rev.pdf.

Engeström, Y. (2007). Enriching the theory of expansive learning: lessons from journeys toward coconfiguration. Mind, Culture and Activity, 14(1-2), 23-39.

. (2009). The future of activity theory: a rough draft. En A. Sannino, H. Daniels y K. Gutiérrez (Eds.). Learning and Expanding with Activity Theory (pp. 303-328). Cambridge: Cambridge University Press.

(2011). From design experiments to formative interventions, Theory \& Psychology, 21(5) 598-628.

Engeström, Y. y Sannino, A. (2016). Expansive learning on the move: insights from ongoing research. Journal for the Study of Education and Development. 39(3), 401-435. doi: https://doi.org/10.108 0/02103702.2016.1189119.

Erausquin, C. y Dome, C. (2016). La investigación-acción como estrategia para re-visitar experiencias, posicionamientos y prácticas ante violencias en escuelas. Anuario de Investigaciones, (23), 87-96. 
Estefoni, C. y Bonhomme, M. (2014). Una vida en Chile y seguir siendo extranjeros. Si Somos Americanos. Revista de Estudios Transfronterizos, 14(2), 81-101.

Fardella, C. y Carvajal, F. (2018). Los estudios sociales de la práctica y la práctica como unidad de estudio. Psicoperspectivas, 17(1). doi: https://doi.org/10.5027/psicoperspectivas-vol17- issue1fulltext-1241.

Fernández, J. y García Castaño, F. (2015). El desarrollo normativo que regula las aulas escolares de nacionalidad extranjera. Profesorado. Revista de currículum y formación del profesorado, 19(1), 468-495.

Flecha, R. (2015). Comunidades de aprendizaje: sueños posibles para todas las niñas y los niños. Aula de Innovación Educativa, (241), 12-16.

Flick, U. (2014). An Introduction to Qualitative Reserch. Berlín: SAGE Publications.

Fraser, N. (2011). Redistribución, reconocimiento y participación. Hacia una concepción integrada de la justicia. En M. Carbonero, y J. Valdivieso (eds.). Dilemas de la Justicia en el siglo XXI. Género y globalización (pp. 291-308). Palma de Mallorca: Edicions Uib.

Fundación Interhumanos. (2018). Resultados Censo docente migración. Santiago: Chile. Recuperado de: http://fundacioninterhumanos.cl/index.php/censo-docente-migracion.

Fundación Superación de la Pobreza, FUSUPO. (2017). Educación e interculturalidad en escuelas públicas. Orientaciones desde la práctica. Santiago: Fundación Superación de la pobreza.

Generalitat de Catalunya. (2015). De l'escola inclusiva al sistema inclusiu. Materials per a l'atenció a la diversitat. Barcelona: Departament D'Ensenyament.

Gentili, P. (2011). Pedagogía de la igualdad. Ensayos contra la educación excluyente. Buenos Aires: Siglo XXI.

González, S. (2017). Las aulas de acogida, la procedencia y la lengua: ¿factores de repliegue relacional en los institutos? Interacções, 13(43), 179-204

González-Falcón, I., Gomez-Hurtado, I., y Álvarez-Diaz, K. (2017). El ATAL como ambiente de aprendizaje inclusivo. Revista del Cisen Tramas/Maepova, 5(1), 141-160.

Harré, R. (2002). Material Objects in Social Worlds. Theory, Culture \& Society, 19(5), 23-33.

Jiménez, R. (2017). Los dispositivos de acogida de los ENAF. Una revisión crítica y comparativa desde una óptica inclusiva, Profesorado. Revista de currículum y formación del profesorado, 21(2), 393-408.

Jiménez, F. y Fardella, C. (2015). Diversidad y rol de la escuela. Discursos del profesorado en contextos educativos multiculturales en clave migratoria, Revista Mexicana de Investigación Educativa, 20(65), 419-441.

Jiménez,F., Aguilera, M., Valdés, R. \& Hernández, M. (2017). Migración y escuela:Análisis documental en torno a la incorporación de inmigrantes al sistema educativo chileno. Psicoperspectivas, 16(1), 105-116. https://dx.doi.org/10.5027/psicoperspectivas-vol16-issue1-fulltext-940.

Kontos, C. (2016). Teoría de la actividad. Aprendizaje expansivo e instrumentos para la reflexión sobre la práctica profesional, interacción alumno-docente y alumno-alumnos, y otros agentes en el aula taller. Escritos en la Universidad, (124), 67-69.

Lamas, M. y Lalueza, J. (2016). Innovar en el aula. Contradicciones entre nuevas herramientas y viejos roles como medio para trasformar la práctica. Estudios Pedagógicos, 42(3), 243-258. doi: http://dx.doi.org/10.4067/S0718-07052016000400013.

Larripa, M. y Erausquin, C. (2008). Teoría de la actividad y modelos mentales. Instrumentos para la reflexión sobre la práctica profesional: "aprendizaje expansivo", intercambio cognitivo y transformación de intervenciones de psicólogos y otros agentes en escenarios educativos, Anuario de Investigaciones, 15, 109-124.

Latour, B. (2008). Reensamblar lo social: una introducción a la teoría del actor-red. Buenos Aires: Manantial.

León, A. (2007). El trueque constructivo: buscando formas respetuosas de trabajo con prácticas contra hegemónicas, Fermentum, 17(50), 626-645.

McDonald, S. (2005). Studying actions in context: A qualitative shadowing method for organizational 
research. Qualitative research, 5(4),455-473. doi: http://dx.doi.org/10.1177/1468794105056923.

Ministerio de Educación. (2017). Orientaciones técnicas para la inclusión educativa de estudiantes extranjeros. Ministerio de Educación: Gobierno de Chile.

(2018). Política nacional de estudiantes extranjeros 2018-2022. Ministerio de Educación: Gobierno de Chile.

Poblete, R. y Galaz, C. (2017). Aperturas y cierres para la inclusión educativa de niños/as migrantes en Chile. Estudios Pedagógicos, 43(3), 239-257. doi: http://dx.doi.org/10.4067/S071807052017000300014.

Pujadas, J. (2018). Etnografía móvil, entre el sombreado y el acompañamiento: notas a partir del estudio de la movilidad cotidiana en la Región Metropolitana de Barcelona (RMB). Etnográfica, 22(2), 361-386. Recuperado de: https://journals.openedition.org/etnografica/5531.

Riedemann, A. (2017). Alumnos inmigrantes en escuelas chilenas: una mirada a las nuevas desigualdades del sistema educativo. En A. Vera (Ed.) Malestar social y desigualdades en Chile. (pp. 99-121). Santiago: Ediciones Universidad Alberto Hurtado.

Rockwell, E. (2009). La experiencia etnográfica. Historia y cultura en los procesos educativos. Buenos Aires: Paidos.

Sannino, A., Engeström, Y. y Lemos, M. (2016). Formative interventions for expansive learning and transformative agency, Journal of the Learning Sciences, 25(4), 599-633.

Simó, N., Pàmies, J., Collet-Sabé, J. y Tort, A. (2014). La acogida educativa en los centros escolares en Cataluña: más allá de los recursos específicos para el alumnado de nueva incorporación, Revista Complutense de Educación, 25(1), 177-194.

Sisto, V. y Zelaya, V. (2013). La Etnografía de Dispositivos y el estudio de los instrumentos de rendición de cuentas como prácticas. Universitas Psychologica, 12(4), 1345-1354. Recuperado de https://revistas.javeriana.edu.co/index.php/revPsycho/article/view/6508.

Valls, R. y Kyriakides, L. (2013). The power of Interactive Groups: how diversity of adults volunteering in classroom groups can promote inclusion and success for children of vulnerable minority ethnic populations. Cambridge Journal of Education, 43(1). doi: 10.1080/0305764X.2012.749213.

Valls, R., Buslón, N. y López, I. (2016). Grupos interactivos: interacciones que aumentan el aprendizaje y la solidaridad. Padres y Maestros, Journal of Parents and Teachers, (367), 17-21.

Vázquez, F. (1996). El análisis de contenido temático. Objetivos y medios en la investigación psicosocial. Documento de trabajo. Universitat Autònoma de Barcelona. Recuperado de: http:// psicologiaysociologia.files.wordpress.com/2013/03/fecc81lixvacc81zquez-sixto_elanacc81lisis-de-contenido-temacc81tico.pdf.

Voirol, J. (2013). ¿Cómo practicar la etnografía? Hacia una teoría pragmática y política de la descripción, Universitas Humanística, (75), 1-24.

Yamazumi, K. (2009). Expansive agency in multi-activity collaboration. En A. Sannino, H. Daniels y K. Gutiérrez (Eds.), Learning and expanding with activity theory, 212-227. 